\title{
INTERPRETATION OF A SHORT-TERM ANOMALY IN THE GRAVITATIONAL MICROLENSING EVENT MOA-2012-BLG-486
}

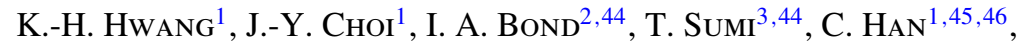

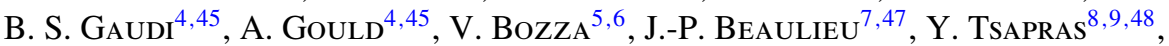
F. Abe ${ }^{10}$, D. P. BennetT ${ }^{11}$, C. S. Botzler ${ }^{12}$, P. Chote ${ }^{13}$, M. Freeman ${ }^{12}$, A. Fukui ${ }^{14}$, D. Fukunaga ${ }^{10}$, P. Harris ${ }^{13}$, Y. Itow $^{10}$, N. Koshimoto ${ }^{3}$, C. H. LinG $^{2}$, K. Masuda ${ }^{10}$, Y. Matsubara ${ }^{10}$, Y. Muraki ${ }^{10}$, S. Namba ${ }^{3}$, K. Ohnishi ${ }^{15}$, N. J. Rattenbury ${ }^{12}$, To. Saito ${ }^{16}$, D. J. Sullivan ${ }^{13}$, W. L. Sweatman ${ }^{2}$, D. Suzuki ${ }^{3}$, P. J. Tristram ${ }^{17}$, K. Wada ${ }^{3}$, N. YAMAI ${ }^{18}$, P. C. M. YocK ${ }^{12}$, A. Yonehara ${ }^{18}$

(The MOA Collaboration)

L. Andrade de Almeida ${ }^{19,20}$, D. L. DePoy ${ }^{21}$, Subo Dong ${ }^{22}$, F. Jablonski ${ }^{19}$, Y. K. Jung ${ }^{1}$, A. Kavka ${ }^{4}$, C.-U. LeE $^{23}$, H. PARK ${ }^{1}$, R. W. PogGE ${ }^{4}$, I.-G. ShIN ${ }^{1}$, J. C. YeE ${ }^{4}$

(The $\mu$ FUN COLlaboration)

M. D. Albrow ${ }^{24}$, E. Bachelet ${ }^{25,26}$, V. Batista ${ }^{7}$, S. Brillant ${ }^{27}$, J. A. R. Caldwell ${ }^{28}$, A. Cassan ${ }^{7}$, A. Cole ${ }^{29}$, E. Corrales ${ }^{7}$, Ch. Coutures ${ }^{7}$, S. Dieters ${ }^{25}$, D. Dominis Prester ${ }^{30}$, J. Donatowicz ${ }^{31}$, P. Fouqué ${ }^{25}, 26$, J. Greenhill ${ }^{29}$, U. G. Jørgensen ${ }^{32}$, S. R. Kane ${ }^{33}$, D. Kubas ${ }^{7,27}$, J.-B. Marquette ${ }^{7}$, R. Martin ${ }^{34}$, P. Meintjes ${ }^{35}$, J. Menzies ${ }^{36}$, K. R. Pollard ${ }^{24}$, A. Williams ${ }^{37}$, D. Wouters ${ }^{7}$

(The PLANET COLlaboration)

D. M. Bramich ${ }^{38,39}$, M. DominiK ${ }^{40,47}$, K. Horne ${ }^{40,47}$, P. Browne ${ }^{40}$, M. Hundertmark ${ }^{40}$, S. IPATOV $^{41}$, N. Kains $^{38}$, C. Snodgrass ${ }^{42}$, I. A. Steele ${ }^{43}$, and R. A. Street $^{8}$

(The Robonet Collaboration)

${ }^{1}$ Department of Physics, Chungbuk National University, Cheongju 361-763, Korea

${ }^{2}$ Institute of Information and Mathematical Sciences, Massey University, Private Bag 102-904, North Shore Mail Centre, Auckland, New Zealand

${ }^{3}$ Department of Earth and Space Science, Osaka University, Osaka 560-0043, Japan

${ }^{4}$ Department of Astronomy, The Ohio State University, 140 West 18th Avenue, Columbus, OH 43210, USA

${ }^{5}$ Dipartimento di Fisica "E. R. Caianiello," Università degli Studi di Salerno, Via S. Allende, I-84081 Baronissi (SA), Italy

${ }^{6}$ Instituto Nazionale di Fisica Nucleare, Sezione di Napoli, I-80126 Napoli, Italy

${ }^{7}$ UPMC-CNRS, UMR7095, Institut d'Astrophysique de Paris, 98bis boulevard Arago, F-75014 Paris, France

${ }^{8}$ Las Cumbres Observatory Global Telescope Network, 6740B Cortona Dr, Goleta, CA 93117, USA

${ }^{9}$ School of Physics and Astronomy, Queen Mary University of London, Mile End Road, London E1 4NS, UK

${ }^{10}$ Solar-Terrestrial Environment Laboratory, Nagoya University, Nagoya 464-8601, Japan

${ }^{11}$ Department of Physics, University of Notre Dame, 225 Nieuwland Science Hall, Notre Dame, IN 46556-5670, USA

12 Department of Physics, University of Auckland, Private Bag 92-019, Auckland 1001, New Zealand

${ }^{13}$ School of Chemical and Physical Sciences, Victoria University, Wellington, New Zealand

${ }^{14}$ Okayama Astrophysical Observatory, National Astronomical Observatory of Japan, Asakuchi, Okayama 719-0232, Japan

${ }_{15}$ Nagano National College of Technology, Nagano 381-8550, Japan

${ }^{16}$ Tokyo Metropolitan College of Aeronautics, Tokyo 116-8523, Japan

${ }^{17}$ Mt. John University Observatory, P.O. Box 56, Lake Tekapo 8770, New Zealand

${ }^{18}$ Department of Physics, Faculty of Science, Kyoto Sangyo University, 603-8555 Kyoto, Japan

${ }^{19}$ Instituto Nacional de Pesquisas Espaciais, São José dos Campos, SP, Brazil

${ }^{20}$ Instituto de Astronomia, Geofísica e Ciências Atmosféricas, IAG/USP Rua do Matão, 1226, Cidade Universitária, São Paulo-SP, Brazil

${ }^{21}$ Department of Physics and Astronomy, Texas A\&M University, College Station, TX 77843, USA

${ }^{22}$ Institute for Advanced Study, Einstein Drive, Princeton, NJ 08540, USA

${ }^{23}$ Korea Astronomy and Space Science Institute, Daejeon 305-348, Korea

${ }^{24}$ Department of Physics and Astronomy, University of Canterbury, Private Bag 4800, 8020 Christchurch, New Zealand

${ }^{25}$ Université de Toulouse, UPS-OMP, IRAP, F-31400 Toulouse, France

${ }^{26}$ CNRS, IRAP, 14 Avenue Edouard Belin, F-31400 Toulouse, France

${ }^{27}$ European Southern Observatory (ESO), Alonso de Cordova 3107, Casilla 19001, Santiago 19, Chile

${ }^{28}$ McDonald Observatory, 16120 St Hwy Spur 78 \#2, Fort Davis, TX 79734, USA

${ }^{29}$ School of Mathematics and Physics, University of Tasmania, Private Bag 37, GPO Hobart, 7001 Tasmania, Australia

${ }^{30}$ Physics Department, Faculty of Arts and Sciences, University of Rijeka, Omladinska 14, 51000 Rijeka, Croatia

${ }^{31}$ Department of Computing, Technical University of Vienna, Wiedner Hauptstrasse 10, A-1040 Vienna, Austria

32 Niels Bohr Institute, Astronomical Observatory, Juliane Maries vej 30, DK-2100 Copenhagen, Denmark

${ }^{33}$ Department of Physics \& Astronomy, San Francisco State University, 1600 Holloway Avenue, San Francisco, CA 94132, USA

${ }^{34}$ Perth Observatory, Walnut Road, Bickley, 6076 Perth, Australia

${ }^{35}$ Faculty of Natural and Agricultural Sciences, Department of Physics, University of the Free State, P.O. Box 339, 9300 Bloemfontein, South Africa

${ }^{36}$ South African Astronomical Observatory, P.O. Box 9, Observatory 7935, South Africa

${ }^{37}$ Astronomisches Rechen-Institut, Zentrum für Astronomie der Universität Heidelberg (ZAH), Mönchhofstr. 12-14, D-69120 Heidelberg, Germany

${ }^{38}$ European Southern Observatory, Karl-Schwarzschild-Street 2, D-85748 Garching bei München, Germany

${ }^{39}$ Qatar Environment and Energy Research Institute, Qatar Foundation, Tornado Tower, Floor 19, P.O. Box 5825, Doha, Qatar

${ }^{40}$ SUPA, School of Physics \& Astronomy, University of St Andrews, North Haugh, St Andrews KY16 9SS, UK

${ }^{41}$ Alsubai Establishment for Scientific Studies, P.O. Box 201536, Doha, Qatar

42 Max Planck Institute for Solar System Research, Max-Planck-Street 2, D-37191 Katlenburg-Lindau, Germany

${ }^{43}$ Astrophysics Research Institute, Liverpool John Moores University, Liverpool CH41 1LD, UK

Received 2013 August 27; accepted 2013 October 1; published 2013 November 1 


\begin{abstract}
A planetary microlensing signal is generally characterized by a short-term perturbation to the standard single lensing light curve. A subset of binary-source events can produce perturbations that mimic planetary signals, thereby introducing an ambiguity between the planetary and binary-source interpretations. In this paper, we present the analysis of the microlensing event MOA-2012-BLG-486, for which the light curve exhibits a short-lived perturbation. Routine modeling not considering data taken in different passbands yields a best-fit planetary model that is slightly preferred over the best-fit binary-source model. However, when allowed for a change in the color during the perturbation, we find that the binary-source model yields a significantly better fit and thus the degeneracy is clearly resolved. This event not only signifies the importance of considering various interpretations of short-term anomalies, but also demonstrates the importance of multi-band data for checking the possibility of false-positive planetary signals.
\end{abstract}

Key words: binaries: general - gravitational lensing: micro - planets and satellites: detection

Online-only material: color figures

\section{INTRODUCTION}

Microlensing is one of the major methods of detecting and characterizing extrasolar planets. The method is important for the comprehensive understanding of planet formation because it is sensitive to planets that are difficult for other methods to detect, especially planets with masses down to an Earth mass located near and beyond the snow line (Sumi et al. 2011; Cassan et al. 2012; Gaudi 2012).

A planetary microlensing signal is characterized by a shortterm perturbation to the standard single lensing light curve. The duration of the signal ranges from several hours for an Earthmass planet to several days for a giant planet. The magnitude of the signal depends weakly on the planet mass although the signal eventually becomes weak for a very low mass planet due to finite-source effects (Bennett \& Rhie 1996). It is known that such perturbations in microlensing light curves can be caused by scenarios other than planetary companions. In particular, some subset of nearly equal-mass binary lenses can exhibit perturbations with similar magnitudes and durations as those caused by very low mass ratio companions (Albrow et al. 2002; Han \& Gaudi 2008). Another example is when the lens is an isolated star, but the source is itself a binary with a large flux ratio. In this case, if the lens passes close to both members of the binary source, the light curve can appear as a normal singlelens curve, superposed with a short-duration perturbation that results when the lens passes close to the fainter source (Gaudi 1998; Gaudi \& Han 2004). Gaudi (1998) pointed out that the frequency of planetary-like perturbations produced by binarysource events can be as high as planet-induced perturbations. Therefore, the analysis of a short-term signal in a lensing light curve requires careful examination considering all possible interpretations.

In this paper, we present the analysis of MOA-2012-BLG486. The light curve of the event exhibits a short-term perturbation. In Section 2, we describe the observation of the event. In Section 3, we describe the analyses conducted to examine the nature of the perturbation and present results. We summarize and conclude in Section 4.

\footnotetext{
${ }^{44}$ The MOA Collaboration.

${ }^{45}$ The $\mu$ FUN Collaboration.

${ }^{46}$ Corresponding author.

${ }^{47}$ The PLANET Collaboration

48 The RoboNet Collaboration.
}

\section{OBSERVATIONS AND DATA}

The microlensing event MOA-2012-BLG-486 occurred on a star in the Galactic bulge field at the equatorial and Galactic coordinates (R.A., decl.) $\mathrm{J}_{2000.0}=\left(18^{\mathrm{h}} 01^{\mathrm{m}} 08^{\mathrm{s}} .82,-33^{\circ} 13^{\prime} 06^{\prime \prime} .1\right)$ and $(l, b)=\left(357.99^{\circ},-5.03^{\circ}\right)$, respectively. It was discovered by the Microlensing Observations in Astrophysics (MOA; Bond et al. 2001; Sumi et al. 2003) survey during the 2012 observing season. From the preliminary analysis of the event based on the rising part of the light curve, it was found that the event would reach a high magnification. A planetary lens system induces a caustic near the position of the primary and thus high-magnification events resulting from the close sourceprimary encounter have a high chance to produce planetary signals (Griest \& Safizadeh 1998). Due to the high sensitivity to planets of the event, an alert was issued for intensive follow-up observations. Based on the alert, the event was additionally observed by other groups including Microlensing Follow-Up Network ( $\mu$ FUN; Gould et al. 2006), Probing Lensing Anomalies NETwork (PLANET; Beaulieu et al. 2006), and RoboNet (Tsapras et al. 2009). In Table 1, we list the survey and follow-up groups along with the telescopes used for observations. We note that although most data were taken in $I$ band, some data sets were obtained in other bands. Realtime modeling played an important role in the acquisition of the color data that ultimately allowed us to distinguish between binary-lens and binary-source models. The Cerro Tololo InterAmerican Observatory (CTIO) VIH points over the first peak were taken as part of normal $\mu$ FUN observing strategy (possible high-magnification event), but the later observations, which measured the colors of the second peak, were taken in direct response to circulation of two models (binary-lens and binarysource), in a specific effort to distinguish them. The event did not return to its baseline brightness until the end of the 2012 bulge season. For secure measurement of the baseline, the event was additionally observed in 2013 season.

Figure 1 shows the light curve of MOA-2012-BLG-486. It is characterized by a short-term anomaly at $\mathrm{HJD}^{\prime}=\mathrm{HJD}-$ $2450000 \sim 6137$. Apart from the anomaly, the overall light curve is well described by the standard form of a single-lens event. Since the characteristics of the light curve strongly indicate the possible existence of a planetary companion, we conduct a detailed analysis of the event.

For the data sets used in the analysis, initial reductions were conducted using photometry codes developed by the individual groups. For some data sets (MOA, CTIO, and South African 
Table 1

Telescopes

\begin{tabular}{lcr}
\hline \hline Group & Telescope & Passband \\
\hline MOA & $1.8 \mathrm{~m}$ Mt. John Observatory, New Zealand & MOA-red \\
$\mu$ FUN & $1.3 \mathrm{~m}$ SMARTS, Cerro Tololo Inter-American Observatory (CTIO), Chile & $V, I, H$ \\
$\mu$ FUN & 0.6 m Observatorio do Pico dos Dias (OPD), Brazil & $I$ \\
PLANET & $1.0 \mathrm{~m}$ South African Astronomical Observatory (SAAO), South Africa & $I$ \\
RoboNet & 2.0 m Faulkes North Telescope (FTN), Hawaii, USA & $I$ \\
RoboNet & 2.0 m Faulkes South Telescope (FTS), Australia & $I$ \\
\hline
\end{tabular}

Note. MOA-red band is a custom wide band where the band width roughly corresponds to the sum of $R$ and $I$ bands.

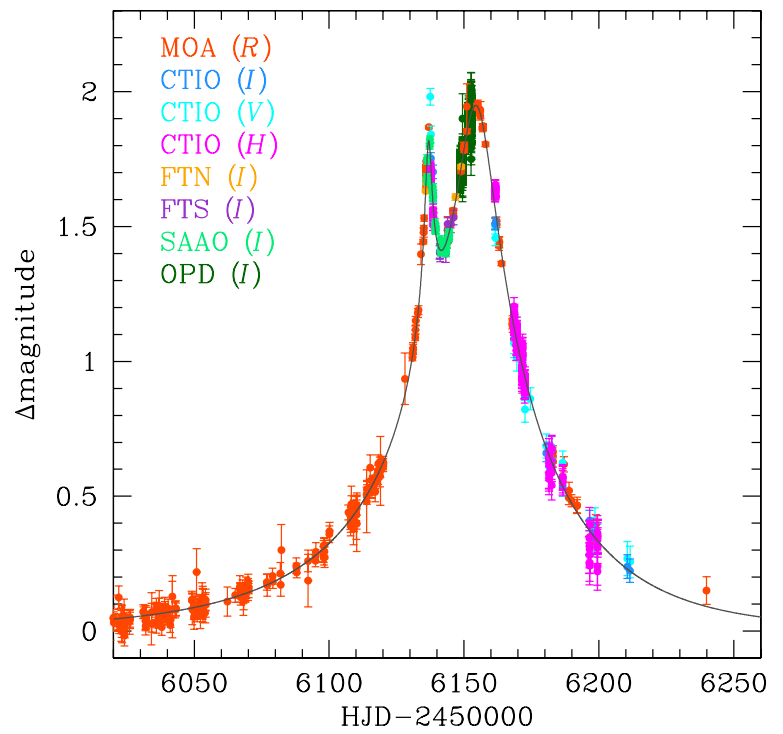

Figure 1. Light curve of MOA-2012-BLG-486. The presented model curve is based on the single-band binary-source model. The notation in the parenthesis after the legend of each observatory denotes the passband of observation.

(A color version of this figure is available in the online journal.)

Astronomical Observatory (SAAO)), we conducted additional reduction to improve photometry using a pipeline based on difference image analysis (Bond et al. 2001; Bramich 2008; Albrow et al. 2009). Nevertheless, the photometric accuracy is limited because there exists a bright star close to the source with a separation $\sim 2$ '.5; see Figure 2 . When this occurs, the photometric measurements are often correlated with the seeing. To minimize the seeing effect, we use data taken with seeing less than $3^{\prime \prime}$.

Since analysis is conducted based on data sets collected from different observatories, we normalized error bars. Error bars of each data set should roughly correspond to the dispersion of the data points at a given time, but this is often not the case due to systematics in a photometry system. Furthermore, error bars estimated by different photometry systems may vary. We normalize the photometry uncertainties of each data set by first adding a quadratic term so that the cumulative distribution of $\chi^{2}$ ordered by magnification is approximately linear and subsequently rescaling the errors so that $\chi^{2}$ per degree of freedom $\left(\chi^{2} /\right.$ dof $)$ for each data set becomes unity (Bachelet et al. 2012; Miyake et al. 2012). The first process ensures the dispersion of data points consistent with error bars regardless of the source brightness (here magnification). The latter process prevents each data set from being under or overweighted compared to other data sets.

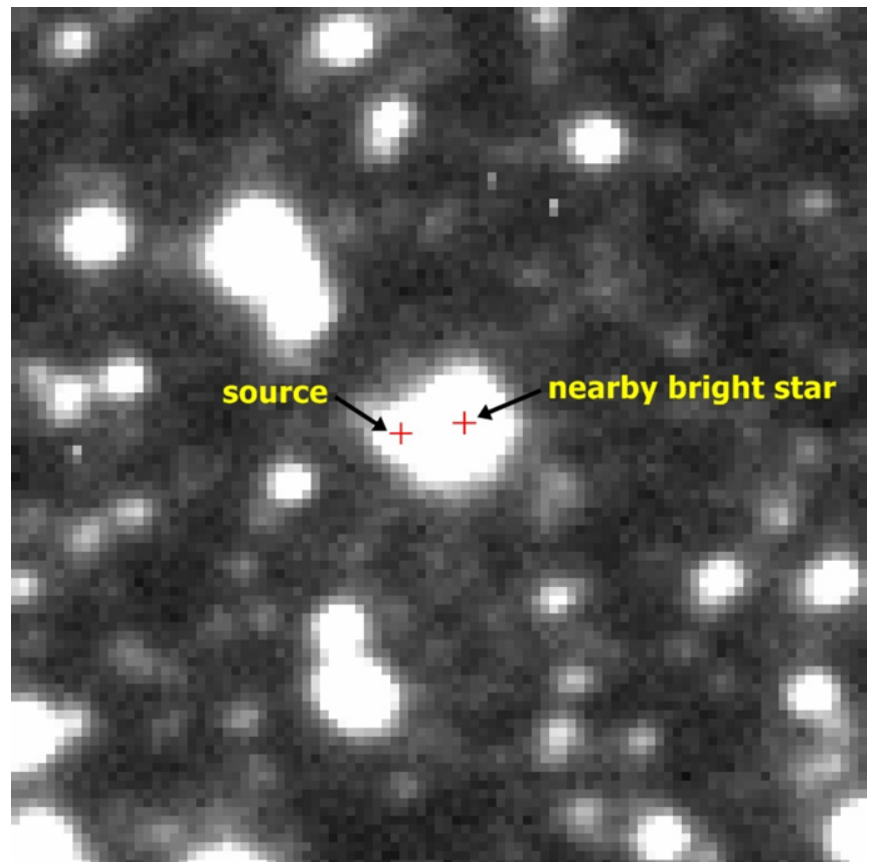

Figure 2. CTIO image of the field around the source star of the microlensing event MOA-2012-BLG-486. The magnification of the source star at the image is $A=2.8$.

(A color version of this figure is available in the online journal.)

\section{MODELING}

Knowing that a short-term perturbation in a lensing light curve can be produced either by a binary (including planetary) companion to the lens or a binary companion to the source, we conduct both binary-lens and binary-source modeling of the light curve. Due to their physical natures, the two types of modeling require widely different parameterization.

Binary lens modeling requires seven basic parameters. Among them, three parameters are needed to describe the lens-source approach, including the time of the closest lens-source approach, $t_{0}$, the lens-source separation at that moment, $u_{0}$ (impact parameter), and the timescale required for the source to cross the Einstein radius of the lens, $t_{\mathrm{E}}$ (Einstein timescale). The Einstein ring represents the image of the source for the case of exact lens-source alignment and its radius $\theta_{\mathrm{E}}$ (Einstein radius) is commonly used as a length scale in lensing phenomena. We note that the lens-source impact parameter $u_{0}$ is normalized by $\theta_{\mathrm{E}}$. Three additional parameters are needed to characterize the star-planet system, including the projected separation, $s$ (normalized by $\theta_{\mathrm{E}}$ ), the mass ratio, $q$, and the source trajectory angle with respect to the binary axis, $\alpha$. A planetary 
perturbation usually occurs when the source encounters a caustic, and so finite-source effects become important. To account for these effects, one needs an additional parameter $\rho_{*}=\theta_{*} / \theta_{\mathrm{E}}$ (normalized source radius), where $\theta_{*}$ represents the angular radius of the source star.

The lensing magnification of a binary-source event corresponds to the flux-weighted mean of the single-lens magnifications associated with the individual source stars, i.e., $A=\left(A_{1} F_{\mathrm{S}, 1}+A_{2} F_{\mathrm{S}, 2}\right) /\left(F_{\mathrm{S}, 1}+F_{\mathrm{S}, 2}\right)$ (Griest \& Hu 1992). Here $F_{\mathrm{S}, i}$ and $A_{i}(i=1,2)$ represent the flux and magnification of the event involved with each source star. To describe the lens approach to the individual source stars, one needs pairs of the impact parameters $\left(u_{0,1}\right.$ and $\left.u_{0,2}\right)$ and times of closest approach $\left(t_{0,1}\right.$ and $\left.t_{0,2}\right)$. Furthermore, an additional parameter is needed to specify the flux ratio between the source stars, $q_{\mathrm{F}}=F_{\mathrm{S}, 2} / F_{\mathrm{S}, 1}$. In our modeling, we set $F_{\mathrm{S}, 1}>F_{\mathrm{S}, 2}$ and thus $q_{\mathrm{F}}<1.0$.

Besides the basic parameters, it is often necessary to consider higher-order effects to precisely describe lensing light curves. The event MOA-2012-BLG-486 lasted for two observing seasons. For such a long timescale event, the positional change of an observer caused by the Earth's orbital motion around the Sun might cause a deviation in the lensing light curve due to the deviation of the apparent lens-source motion from a rectilinear trajectory (Gould 1992). Considering this parallax effect requires two additional lensing parameters $\pi_{\mathrm{E}, N}$ and $\pi_{\mathrm{E}, E}$, which are the components of the lens parallax vector $\boldsymbol{\pi}_{\mathrm{E}}$ projected on the sky along the northern and eastern equatorial axes, respectively. For the binary-lens case, the positional change of the lens caused by the orbital motion can also induce long-term deviations in lensing light curves. To first-order approximation, the lens orbital effect is described by the two parameters $d s / d t$ and $d \alpha / d t$ that represent the change rates of the normalized binary separation and source trajectory angle, respectively (Albrow et al. 2000; An et al. 2002).

For both binary-lens and binary-source models, we look for best-fit sets of lensing parameters by running a Markov Chain Monte Carlo search of the parameter space. In the initial binary-source modeling, we model the light curve with a single flux ratio (single-band model). In Table 2, we list the best-fit parameters for the individual models. We find that the overall shape and the short-term feature of the light curve can be described by both planetary and binary-source models with similar values of $\chi^{2}: \chi^{2}=2828.8$ for the planetary model and $\chi^{2}=2855.7$ for the binary-source model (single band). However, there exist some residuals from the models, especially in the region of the short-term anomaly as shown in the lower panels of Figure 3. Although consideration of parallax and orbital (for the planetary model) effects somewhat improves the fits of both models, they contribute to the fits of the overall shape of the light curve, not to the anomaly feature. This indicates the need to consider another higher-order effect.

Color change can occur for a binary-source event. This color change occurs because the stars comprising a binary source have, in general, different colors and thus the colors measured at the moments of the lens' approaches close to the individual source stars can be different. From the initial single-band binarysource modeling, the measured flux ratio between the two source stars, $q_{\mathrm{F}} \sim 0.1$, is small. This implies that the types of the source stars are likely to be very different and thus color effects would be pronounced during the perturbation. To check whether the color effect can explain the residual in the anomaly, we conduct additional binary-source modeling. For this, we specify flux ratios for the individual passbands of the data covering the

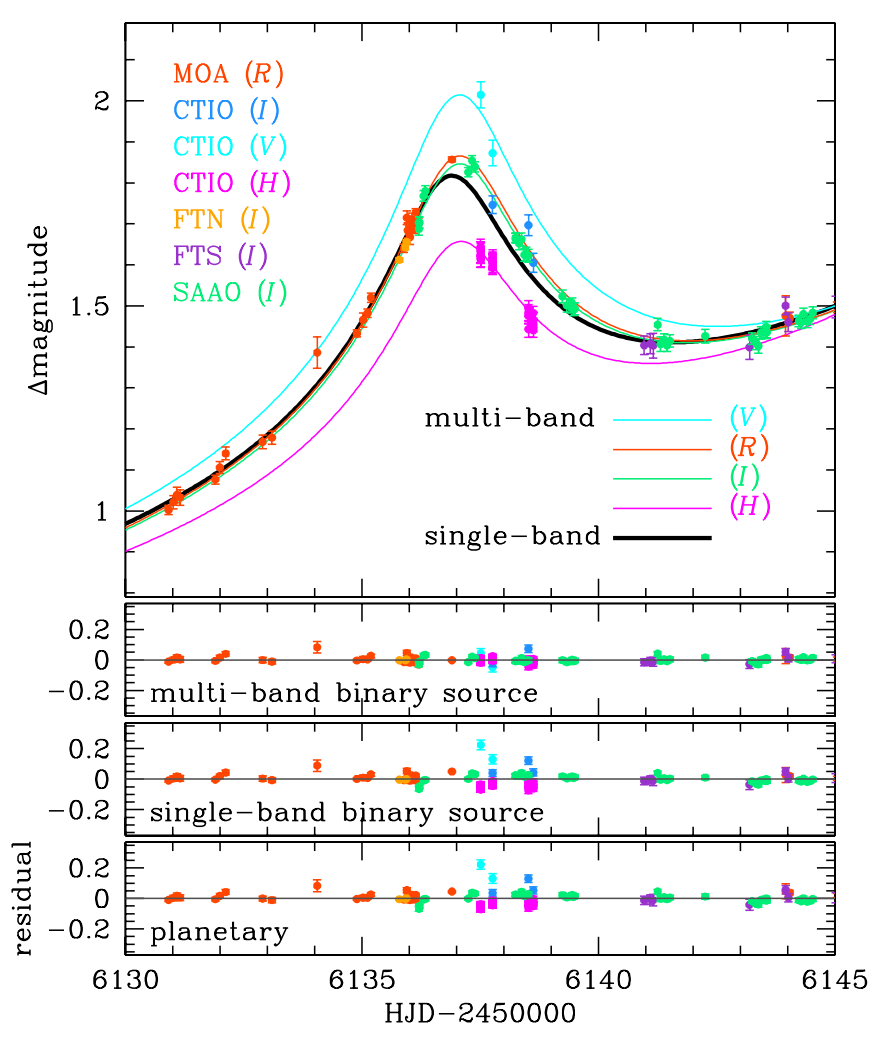

Figure 3. Zoom of the perturbation region of the light curve of MOA-2012-BLG486. The thick solid black curve is the best-fit model obtained from single-band binary-source modeling, while thin curves with various colors are model curves considering color effects. The three bottom panels show the residuals from the three sets of modeling.

(A color version of this figure is available in the online journal.)

anomaly, including $V$ (CTIO), $R$ (MOA), $I$ (CTIO, Faulkes North Telescope (FTN), Faulkes South Telescope (FTS), SAAO), and $H$ (CTIO) band.

In Figure 3, we present the light curves in the region of the anomaly resulting from modeling considering the color effect (multi-band binary-source model). We note that since lensing magnifications depend on the observed passband, four model light curves corresponding to the individual passbands are presented. The measured flux ratio of each passband between the binary-source stars is listed in Table 2 . We find that the model considering the color effect significantly improves the fit with $\Delta \chi^{2}=690.4$ compared to the single-band binary-source model. Furthermore, the multi-band binary-source model is better than the planetary model by $\Delta \chi^{2}=663.5$. The improvement of the fit can be visually seen from the residuals presented in Figure 3 . The superiority of the fit compared to the planetary model combined with the obvious color effect strongly supports the binary-source interpretation of the anomaly.

In Figure 4, we present the trajectories of the individual source stars with respect to the lens for the best-fit binary-source model. The fact that the event is brighter in shorter wavelength passbands during the anomaly indicates that the faint source component (secondary) is bluer than the bright component (primary). We choose the color of each source trajectory in order to reflect the color of each star. According to the model, the faint blue star approached very close to the lens, producing a shortterm anomaly, and then the bright red star followed. The flux ratio between source stars is small, ranging from 0.08 ( $H$ band) to 0.16 ( $V$ band). As a result, the light curve is dominated by 
Table 2

Lensing Parameters

\begin{tabular}{|c|c|c|c|}
\hline \multirow[t]{2}{*}{ Parameters } & \multirow[t]{2}{*}{ Planetary } & \multicolumn{2}{|c|}{ Binary Source } \\
\hline & & Single Band & Multi Bands \\
\hline$\chi^{2} /$ dof & $2828.8 / 2184$ & $2855.7 / 2187$ & 2165.3/2184 \\
\hline$t_{0,1}\left(\mathrm{HJD}^{\prime}\right)$ & $6149.33 \pm 0.24$ & $6154.43 \pm 0.02$ & $6154.24 \pm 0.03$ \\
\hline$t_{0,2}\left(\mathrm{HJD}^{\prime}\right)$ & $\ldots$ & $6137.19 \pm 0.05$ & $6137.20 \pm 0.05$ \\
\hline$u_{0,1}$ & $0.059 \pm 0.002$ & $0.099 \pm 0.004$ & $0.077 \pm 0.003$ \\
\hline$u_{0,2}$ & $\ldots$ & $0.024 \pm 0.003$ & $0.027 \pm 0.002$ \\
\hline$t_{\mathrm{E}}$ (days) & $92.8 \pm 2.2$ & $66.4 \pm 1.9$ & $78.1 \pm 2.8$ \\
\hline$s$ & $1.65 \pm 0.01$ & $\ldots$ & $\ldots$ \\
\hline$q$ & $0.029 \pm 0.001$ & $\ldots$ & $\ldots$ \\
\hline$\alpha$ & $2.789 \pm 0.006$ & $\ldots$ & $\ldots$ \\
\hline$\rho_{*}\left(10^{-3}\right)$ & $0.5 \pm 0.3$ & $\ldots$ & $\ldots$ \\
\hline$\pi_{\mathrm{E}, N}$ & $-0.51 \pm 0.04$ & $-0.18 \pm 0.11$ & $-0.37 \pm 0.09$ \\
\hline$\pi_{\mathrm{E}, E}$ & $0.11 \pm 0.03$ & $0.17 \pm 0.03$ & $0.08 \pm 0.02$ \\
\hline$d s / d t$ & $-1.62 \pm 0.28$ & $\ldots$ & $\ldots$ \\
\hline$d \alpha / d t$ & $-0.46 \pm 0.13$ & $\ldots$ & $\ldots$ \\
\hline$q_{F}$ & $\ldots$ & $0.097 \pm 0.002$ & $\ldots$ \\
\hline$q_{\mathrm{F}, V}$ & $\ldots$ & $\ldots$ & $0.163 \pm 0.007$ \\
\hline$q_{\mathrm{F}, R}$ & $\ldots$ & $\ldots$ & $0.125 \pm 0.003$ \\
\hline$q_{\mathrm{F}, I}$ & $\ldots$ & $\ldots$ & $0.118 \pm 0.003$ \\
\hline$q_{\mathrm{F}, H}$ & $\ldots$ & $\ldots$ & $0.081 \pm 0.002$ \\
\hline
\end{tabular}

Note. HJD' $=$ HJD -2450000 .

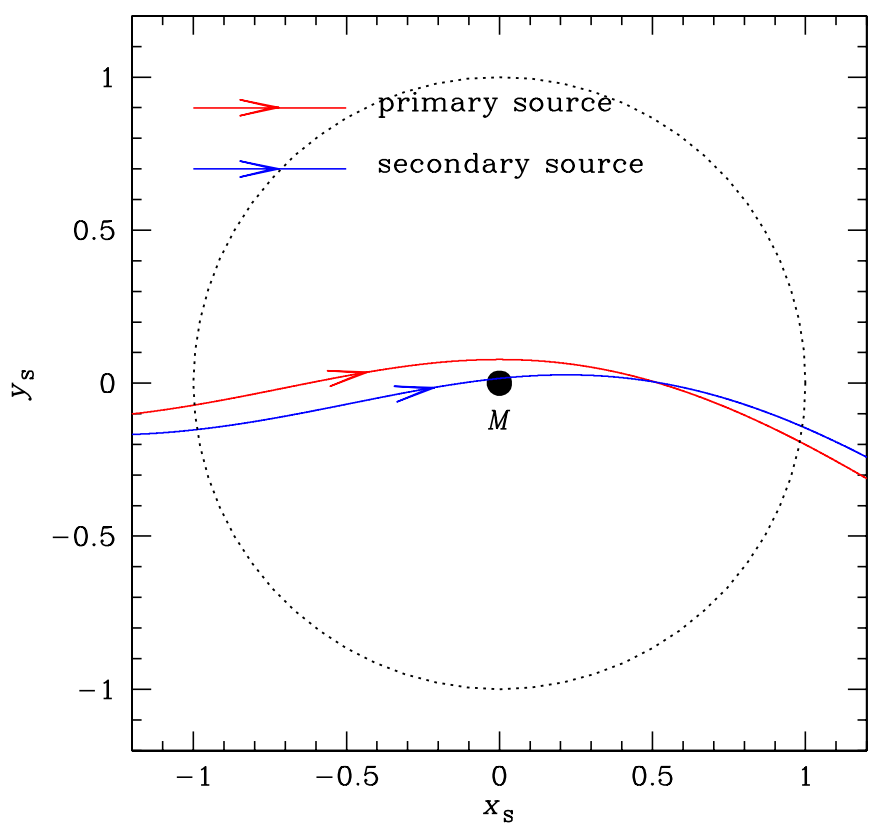

Figure 4. Geometry of the lens system for the binary-source model. The lens is positioned at the origin (marked by $M$ ) and the dotted circle is the Einstein ring. The two curves with arrows represent the trajectories of the two source stars. Lengths are scaled by the Einstein radius.

(A color version of this figure is available in the online journal.)

the light from the bright source star except for the short term of the lens' approach to the faint source star. We note that the trajectories are curved due to parallax effects.

In Figure 5, we present the locations of the binary-source components in the color-magnitude diagram. Based on the source flux measured from modeling combined with the reference position of the centroid of clump giants with the known de-reddened magnitude at the Galactic distance $I_{0}=14.54$ (Nataf et al. 2013) and color $(V-I)_{0}=1.06$ (Bensby et al. 2011), we determine the de-reddened magnitude and color of the individual source stars

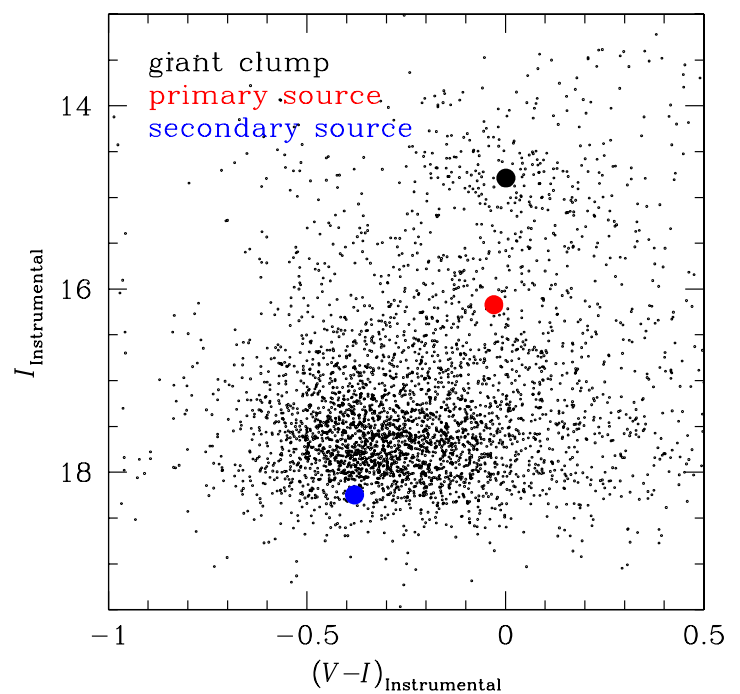

Figure 5. Positions of the components stars of the binary source in the color-magnitude diagram.

(A color version of this figure is available in the online journal.)

as $(I, V-I)_{0}=(15.93,1.03)$ for the primary and $(18.25,0.68)$ for the secondary. These correspond to a K-type giant and a turn-off star for the primary and secondary, respectively.

We check the possibility of the limb-darkening surface brightness variation of the source star as a cause of the color variation. From additional modeling, we find that $\chi^{2}$ improvement by the limb-darkening effect is negligible and thus exclude the possibility.

\section{CONCLUSION}

We analyzed the microlensing event MOA-2012-BLG-486 where the light curve exhibited a short-term perturbation indicating the possible existence of a planetary companion to the lens. By conducting detailed modeling of the light curve considering both planetary and binary-source interpretations, 
we found that the perturbation was better explained by the binary-source model. The degeneracy between the planetary and binary-source interpretations was clearly resolved via the color effect that occurred during the anomaly due to the multi-band data obtained during the anomaly. The event not only signifies the importance of considering various interpretations of shortterm anomalies but also demonstrates the importance of multiband data for checking the possibility of false-positive planetary signals.

Our ability to distinguish between the planetary and binarysource solutions rested critically on $V I H$ photometry. However, while such dense multi-band photometry is routine for follow-up observations from $\mu$ FUN SMARTS CTIO, survey observations typically take data overwhelmingly in one band. With the advent of second generation surveys, a large fraction of microlensing planets are being detected from survey-only data, and this will be increasingly true in the future. Hence, the protocols for these surveys should be carefully evaluated to make sure that color coverage is adequate to distinguish binary-source events from planetary events.

Work by C.H. was supported by Creative Research Initiative Program (2009-0081561) of National Research Foundation of Korea. The MOA experiment was supported by grants JSPS22403003 and JSPS23340064. T.S. acknowledges the support JSPS 24253004. T.S. is supported by the grant JSPS23340044. Y.M. acknowledges support from JSPS grants JSPS23540339 and JSPS19340058. A.G. and B.S.G. acknowledge support from NSF AST-1103471. B.S.G., A.G., and R.W.P. acknowledge support from NASA grant NNX12AB99G. S.D. was supported through a Ralph E. and Doris M. Hansmann Membership at the IAS and NSF grant AST-0807444. D.M.B.,
M.D., K.H., M.H., S.I., C.S., R.A.S., and Y.T. are supported by NPRP grant NPRP-09-476-1-78 from the Qatar National Research Fund (a member of Qatar Foundation). K.H. is supported by a Royal Society Leverhulme Senior Research Fellowship. C.S. received funding from the European Union Seventh Framework Programme (FP7/2007-2013) under grant agreement no. 268421. The research leading to these results has received funding from the European Community's Seventh Framework Programme (/FP7/2007-2013/) under grant agreement No 229517.

\section{REFERENCES}

Albrow, M. D., An, J., Beaulieu, J.-P., et al. 2002, ApJ, 572, 1031 Albrow, M. D., Beaulieu, J.-P., Caldwell, J. A. R., et al. 2000, ApJ, 534, 894 Albrow, M. D., Horne, K., Bramich, D. M., et al. 2009, MNRAS, 397, 2099 An, J. H., Albrow, M. D., Beaulieu, J.-P., et al. 2002, ApJ, 572, 521 Bachelet, E., Fouqué, P., Han, C., et al. 2012, A\&A, 547, A55 Beaulieu, J.-P., Bennett, D. P., Fouqué, P., et al. 2006, Natur, 439, 437 Bennett, D. P., \& Rhie, S. H. 1996, ApJ, 472, 660

Bensby, T., Adén, D., Meléndez, J., et al. 2011, A\&A, 533, A134 Bond, I. A., Abe, F., Dodd, R. J., et al. 2001, MNRAS, 327, 868 Bramich, D. M. 2008, MNRAS, 386, L77

Cassan, A., Kubas, D., Beaulieu, J.-P., et al. 2012, Natur, 481, 167

Gaudi, B. S. 1998, ApJ, 506, 533

Gaudi, B. S. 2012, ARA\&A, 50, 411

Gaudi, B. S., \& Han, C. 2004, ApJ, 611, 528

Gould, A. 1992, ApJ, 392, 442

Gould, A., Udalski, A., An, D., et al. 2006, ApJL, 644, L37

Griest, K., \& Hu, W. 1992, ApJ, 397, 362

Griest, K., \& Safizadeh, N. 1998, ApJ, 500, 37

Han, C., \& Gaudi, B. S. 2008, ApJ, 689, 53

Miyake, N., Udalski, A., Sumi, T., et al. 2012, ApJ, 752, 82

Nataf, D. M., Gould, A., Fouqué, P., et al. 2013, ApJ, 769, 88

Sumi, T., Abe, F., Bond, I. A., et al. 2003, ApJ, 591, 204

Sumi, T., Kamiya, K., Bennett, D. P., et al. 2011, Natur, 473, 349

Tsapras, Y., Street, R., Horne, K., et al. 2009, AN, 330, 4 